COCHRANE CORNER

${ }^{\dagger}$ This review is an abridged version of a Cochrane review previously published in the Cochrane Database of Systematic Reviews, 2018, December 20, Issue 12: CD007964 (doi: 10.1002/14651858.CD007964. pub2) (see www.Cochranelibrary.com for information). Cochrane reviews are regularly updated as new evidence emerges and in response to feedback, and the Cochrane Database of Systematic Reviews should be consulted for the most recent version of the review.

(C) 2018 The Cochrane Collaboration. Published by John Wiley \& Sons Ltd.

We thank the Cochrane Review Group for their support in publishing these reviews.

See commentary in this issue.

\title{
Cognitive behavioural therapy plus standard care versus standard care for people with
} schizophrenia

Christopher Jones, David Hacker, Jun Xia, Alan Meaden, Claire B. Irving, Sai Zhao, Jue Chen \& Chunhu Shi

\section{Background}

Cognitive behavioural therapy (CBT) is a psychosocial treatment that aims to re-mediate distressing emotional experiences or dysfunctional behaviour by changing the way in which a person interprets and evaluates the experience or cognates on its consequence and meaning. This approach helps to link the feelings and patterns of thinking that underpin the person's distress. CBT is now recommended by the National Institute for Health and Care Excellence (NICE) as an add-on treatment for people with a diagnosis of schizophrenia. This review is part of a family of Cochrane CBT reviews for the treatment of people with schizophrenia.

\section{Objectives}

To assess the effects of CBT added to standard care compared with standard care alone for people with schizophrenia.

\section{Search methods}

We searched the Cochrane Schizophrenia Group's Trials Register (up to 6 March 2017). This register is compiled by systematic searches of major resources (including AMED, BIOSIS CINAHL, Embase, MEDLINE, PsycINFO, PubMed, and registries of clinical trials) and their monthly updates, handsearches, grey literature and conference proceedings, with no language, date, document type or publication status limitations for inclusion of records into the register.

\section{Selection criteria}

We selected all randomised controlled clinical trials (RCTs) involving people diagnosed with schizophrenia or related disorders that compared adding CBT to standard care with standard care given alone. Outcomes of interest included relapse, readmission to hospital, mental state, adverse events, social functioning, quality of life and satisfaction with treatment. We included studies fulfilling the predefined inclusion criteria and reporting useable data.

Data collection and analysis

We complied with the Cochrane recommended standard of conduct for data screening and collection. Where possible, we calculated relative risk (RR) and its $95 \%$ confidence interval (CI) for binary data and mean difference (MD) and its $95 \%$ confidence interval for continuous data. We assessed risk of bias for included studies and created a 'Summary of findings' table using GRADE.

\section{Main results}

This review now includes 60 trials with 5992 participants, all comparing CBT added to standard care with standard care alone. Results for the main outcomes of interest (all long term) showed no clear difference between CBT and standard care for relapse ( $\mathrm{RR}=0.78,95 \% \mathrm{Cl} 0.61-1.00 ; 1538$ participants; 13 studies, low-quality evidence). Two trials reported global state improvement. More participants in the CBT groups showed clinically important improvement in global state $(R R=0.57$, 95\% Cl 0.39-0.84; 82 participants; 2 studies, very low-quality evidence). Five trials reported mental state improvement. No differences in mental state improvement were observed $(\mathrm{RR}=0.81$, 95\% Cl 0.65-1.02; 501 participants; 5 studies, very low-quality evidence). In terms of safety, adding CBT to standard care may reduce the risk of having an adverse event $(\mathrm{RR}=0.44,95 \% \mathrm{Cl}$ $0.27-0.72$; participants $=146$; studies $=2$, very low-quality evidence), but it appears to have no effect on long-term social functioning $(\mathrm{MD}=0.56,95 \% \mathrm{Cl}-2.64$ to $3.76 ; 295$ participants; 2 studies, very low-quality evidence) or long-term quality of life (MD $=-3.60,95 \% \mathrm{Cl}-11.32$ to $4.12 ; 71$ participants; 1 study, very low-quality evidence). It also has no effect on long-term satisfaction with treatment (measured as 'leaving the study early') (RR $=0.93,95 \%$ Cl 0.77-1.12; 1945 participants; 19 studies, moderate-quality evidence)

\section{Authors' conclusions}

Relative to standard care alone, adding CBT to standard care appears to have no effect on long-term risk of relapse. A very small proportion of the available evidence indicated that CBT plus standard care may improve long-term global state and may reduce the risk of adverse events. Whether adding CBT to standard care leads to clinically important improvement in patients' long-term mental state, quality of life and social functioning remains unclear. Satisfaction with care (measured as the number of people leaving the study early) was no higher for participants receiving CBT plus standard care compared with participants receiving standard care alone. It should be noted that, although much research has been carried out in this area, the quality of evidence available is poor - mostly low or very low quality - and we still cannot make firm conclusions until more high-quality data are available. 